\title{
Identifying predictors of hepatic disease in patients after the Fontan operation: A postmortem analysis
}

\author{
Jennifer A. Johnson, DO, ${ }^{a}$ Frank Cetta, MD, ${ }^{\text {a,b }}$ Rondell P. Graham, MBBS, ${ }^{\mathrm{c}}$ Thomas C. Smyrk, MD, ${ }^{\mathrm{c}}$ \\ David J. Driscoll, MD, ${ }^{\mathrm{a}, \mathrm{b}}$ Sabrina D. Phillips, MD, ${ }^{\mathrm{b}}$ and Anitha S. John, MD, $\mathrm{PhD}^{\mathrm{a}, \mathrm{b}, \mathrm{d}}$
}

Objective: We characterized the histologic liver changes that occur after the Fontan operation and identified the factors associated with worsening hepatic disease.

\begin{abstract}
Methods: From 1979 to 2009, 33 patients who died after the Fontan operation had a postmortem analysis at our institution. The liver histologic findings were graded for sinusoidal and portal fibrosis.

Results: The median interval from the Fontan operation to death was 14 days (range, 0 days to 25.6 years). The median age at death was 7.7 years (range, 1.9-52.9 years). Hepatic sinusoidal fibrosis occurred in 25 of 33 patients $(76 \%)$, with significant fibrosis (grade 2 or greater) in 15 of 25 patients $(60 \%)$. Portal fibrosis occurred in 17 of 33 patients $(52 \%)$. The degree of sinusoidal $(P<.003)$ and portal fibrosis $(P<.002)$ correlated with an increased period from the Fontan operation to death. Sinusoidal fibrosis was also associated with increased age at the initial Fontan operation $(P<.03)$. Of the patients who died early $(<35$ days postoperatively) after the Fontan operation, sinusoidal fibrosis was present in 15 of the 23 patients $(65 \%)$ and portal fibrosis in 7 of 23 patients $(30 \%)$. Significant (grade 2 or greater) sinusoidal fibrosis was seen in 7 patients, and 4 of the 7 had heterotaxy syndrome. Significant portal fibrosis was observed in only 2 patients; both had heterotaxy syndrome.
\end{abstract}

Conclusions: The interval from the initial Fontan operation to death is associated with increased sinusoidal and portal fibrosis. In this small cohort, older age at Fontan operation was associated with sinusoidal fibrosis. Significant sinusoidal fibrosis was observed in patients who died early after the Fontan operation, with more severe disease present in patients with heterotaxy syndrome. (J Thorac Cardiovasc Surg 2013;146:140-5)

First described in 1971, the Fontan operation is now used widely for patients with single ventricle morphology. ${ }^{1} \mathrm{Nu}-$ merous modifications have been made to the Fontan operation, but the basic principle is to direct systemic venous flow directly to the pulmonary arteries, circumventing the need for a subpulmonary ventricle. A consequence of this circulation is increased systemic venous pressure, which has been associated with increased morbidity and mortality. ${ }^{2,3}$

Late complications after the Fontan operation include arrhythmias, heart failure, thromboemboli, protein-losing enteropathy (PLE), and hepatic dysfunction. ${ }^{4}$ The first description of hepatic fibrosis in a patient after the Fontan operation was in 1981. Since then, several studies have described high-grade hepatic fibrosis and hepatic cirrhosis in this population..$^{5-9}$ In addition, increasing numbers of case

From the Divisions of Pediatric Cardiology, ${ }^{\mathrm{a}}$ and Cardiovascular Diseases, ${ }^{\mathrm{b}}$ and Department of Laboratory Medicine and Pathology, ${ }^{\mathrm{c}}$ Mayo Clinic, Rochester, Minn; and Division of Cardiology, ${ }^{\mathrm{d}}$ Children's National Medical Center, George Washington University, Washington, DC.

Disclosures: Authors have nothing to disclose with regard to commercial support.

Received for publication May 23, 2012; revisions received July 30, 2012; accepted for publication Sept 12, 2012; available ahead of print Oct 15, 2012.

Address for reprints: Frank Cetta, MD, Division of Pediatric Cardiology, Mayo Clinic, 2001 st St, Southwest, Gonda 6335, Rochester, MN 55905 (E-mail: cetta. frank@mayo.edu).

0022-5223/\$36.00

Copyright (C) 2013 by The American Association for Thoracic Surgery

http://dx.doi.org/10.1016/j.jtcvs.2012.09.005 reports have been published of hepatocellular carcinoma occurring in patients after the Fontan operation. ${ }^{10}$

The proposed mechanisms for hepatic injury, with resulting sinusoidal fibrosis, include increased central and portal venous pressure, decreased cardiac output, and flow reversal in the hepatic veins. These changes cause hepatic injury through hypoxia, chronic congestion, and stimulation of the fibrotic response. ${ }^{7}$ A recent study reported portal fibrosis can occur before the Fontan operation from pre-Fontan insults and elevated right atrial pressure. ${ }^{8}$ The aim of the present study was to further characterize the histologic liver changes that occur after the Fontan operation and to identify any factors associated with worsening hepatic disease.

\section{METHODS}

From 1979 to 2009, patients who died after the Fontan operation were identified from existing clinical databases at the Mayo Clinic. Of those patients, 33 patients had had complete autopsies done at the Mayo Clinic. Clinical variables were obtained by retrospective chart review. The extracted variables included patient demographics, cardiac anatomy, surgical details, cause of death, and previous history of liver disease. The Mayo Foundation institutional review board reviewed and approved the protocol (protocol no. 10-004994).

Hematoxylin and eosin-stained autopsy liver slides were retrieved for the identified cases. Formalin-fixed paraffin-embedded tissue blocks were obtained, and $4-\mu \mathrm{m}$ tissue sections were cut. The tissue sections were deparaffinized with xylene and stained with Masson's trichrome. The autopsy slides were independently reviewed by 2 liver pathologists (R.P.G and T.C.S) who were unaware of the patient characteristics and 


\section{Abbreviation and Acronym}

PLE $=$ protein-losing enteropathy

treatment. The following histologic parameters were assessed semiquantitatively: sinusoidal fibrosis and portal fibrosis stage. The portal fibrosis stage in each case was documented according to the most severe areas using the previously published modified Ishak ${ }^{11}$ and Scheuer ${ }^{12}$ scoring systems. In addition, portal venous congestion (as determined by $>2$ portal vein branch profiles in a portal tract or portal vein ectopia), hepatic sinusoidal congestion, and central vein fibrosis and centrilobular necrosis were also semiquantitatively assessed. ${ }^{11,12}$

The data were analyzed using JMP, version 7.0, statistical software (SAS Institute, Cary, NC). Data are expressed as the median and range or mean \pm standard deviation for continuous variables. Proportions were analyzed and compared using Fisher's exact test. Simple linear regression analysis was used to determine whether the patient variables were significantly associated with the histologic grading results. The mean values were analyzed using the independent group $t$ test for mean. A 2-sided $P$ value $<.05$ was considered statistically significant.

\section{RESULTS}

\section{Demographics and Cardiac History}

The patient characteristics are listed in Table 1. Of the 33 deaths, 23 were early ( $<35$ days after the Fontan operation) and 10 were late. The median patient age at the initial Fontan operation was 6.2 years (range, 1.9-35.9 years). The median interval from the Fontan operation to death was 5 days (range, 0-34 days) for the early deaths and 10.1 years (range, 1.7-25.6 years) for the late deaths. The causes of death included multisystem organ failure in $8(27 \%)$, cardiac arrest/failure in $14(47 \%)$, infection in $4(13 \%)$; hemorrhage in $3(9 \%)$, pulmonary embolus in $1(3 \%)$, and unknown in $3(9 \%)$.

The cardiac anatomy included 10 patients (30\%) with tricuspid atresia, $9(28 \%)$ with a double-inlet left ventricle, 5 $(15 \%)$ with a double-outlet right ventricle, $3(9 \%)$ with complete atrioventricular septal defects, $3(9 \%)$ with pulmonary atresia, $1(3 \%)$ with mitral valve atresia, $1(3 \%)$ with Ebstein anomaly, and 1 (3\%) with criss-cross ventricles. The ventricular morphology was left in 23 patients $(70 \%)$ and right in $10(30 \%)$. Three patients who died late after the Fontan operation had PLE. Nine patients $(27 \%)$ had heterotaxy syndrome (six with asplenia and three with polysplenia). Of the 9 patients with heterotaxy syndrome, 7 had died early and 2 late after the Fontan operation. Five patients $(15 \%)$ had a recorded negative hepatitis $\mathrm{C}$ screening result. PLE did not correlate independently with the degree of hepatic fibrosis.

\section{Surgical History}

The types of Fontan connections included atriopulmonary in $28(85 \%)$, right atrium to right ventricle in $2(6 \%)$, lateral tunnel or intra-atrial conduit in $2(6 \%)$, and extracardiac conduit in $1(3 \%)$. The Fontan operations were performed from 1979 to 2004. One patient had a fenestration at the initial Fontan operation. Only 1 patient had a mechanical valve placed in the aortic position, and 1 patient had a mechanical valve placed in the right atrioventricular valve position at the Fontan operation.

Of the 33 patients, 32 had undergone cardiac operations before the Fontan operation. These initial operations are summarized in Table 2. Seven patients had undergone multiple cardiac operations before the Fontan operation. Two patients had undergone a Blalock-Taussig shunt followed by a Glenn anastomosis. Three patients had undergone a Blalock-Taussig shunts followed by central aorticopulmonary shunts.

Three patients ( 1 early death and 2 late deaths) underwent take down of the Fontan connection to either a cavopulmonary anastomosis or an aorticopulmonary shunt. Of the late deaths, 3 patients had undergone Fontan conversions from an atriopulmonary connection to intra-atrial conduits because of refractory arrhythmias, and 1 patient required surgical revision of the right atrial to main pulmonary artery connection owing to obstruction of the Fontan pathway. Two of these patients had undergone removal of thrombus within the Fontan pathway at Fontan conversion.

\section{Histologic Assessment}

Hepatic sinusoidal congestion was observed in 31 of 33 patients $(94 \%)$. Of the 2 patients without sinusoidal congestion, 1 died early after a tricuspid valve repair failed that had led to the need for a Fontan operation. The second patient presented with congestive heart failure and atrial flutter and died of an arrhythmia during transesophageal echocardiography. Centrilobular necrosis occurred 11 of 33 patients $(33 \%)$, and central vein fibrosis occurred in 26 of 33 patients $(79 \%)$. No statistically significant correlations were found between the degree of hepatic congestion, centrilobular necrosis, or central vein fibrosis and patient age at the Fontan operation or interval from the Fontan operation to death $(P=.06)$. Portal tract venous congestion occurred in 25 of 33 patients $(76 \%)$. The degree of portal congestion varied inversely with the interval from the Fontan operation to death $(P<.007$; Figure $1, B)$. Of the 8 patients without portal tract venous congestion, 6 had undergone the Fontan operation more than 7.4 years previously.

\section{Sinusoidal Fibrosis}

Sinusoidal fibrosis occurred in 25 of 33 patients $(76 \%)$, with significant sinusoidal fibrosis (grade 2 or greater) observed in 15 of 25 patients $(60 \%$; Table 3 and Figure 2, $B$ and $D$ ). The interval from Fontan operation to death varied greatly in these patients (range, 6 days to 25.6 years). The degree of sinusoidal fibrosis correlated with increased time from the Fontan operation (univariate, $P<.003$; Figure $1, A)$ and increased age at the initial Fontan operation (univariate, $P<.03$ ). Of the 5 patients with grade 4 
TABLE 1. Cohort description

\begin{tabular}{|c|c|c|c|}
\hline Variable & $\begin{array}{c}\text { Early deaths } \\
\quad(n=23)\end{array}$ & $\begin{array}{l}\text { Late deaths } \\
\qquad(\mathbf{n}=10)\end{array}$ & $\begin{array}{l}\text { Total deaths } \\
\quad(\mathbf{n}=\mathbf{3 3})\end{array}$ \\
\hline \multicolumn{4}{|l|}{ Age at Fontan operation (y) } \\
\hline Median & 4.7 & 13.1 & 6.2 \\
\hline Range & $1.9-35.9$ & $4.3-34.5$ & $1.9-35.9$ \\
\hline \multicolumn{4}{|l|}{ Age at death (y) } \\
\hline Median & 4.7 & 17.1 & 7.7 \\
\hline Range & $1.9-36.0$ & $10.3-52.9$ & $1.9-52.9$ \\
\hline \multicolumn{4}{|l|}{$\begin{array}{l}\text { Interval from Fontan } \\
\quad \text { operation to death (d) }\end{array}$} \\
\hline Median & 5 & 10.1 & $14 \mathrm{~d}$ \\
\hline Range & $0-34$ & $1.7-25.6$ & $0 \mathrm{~d}$ to $25.6 \mathrm{y}$ \\
\hline \multicolumn{4}{|l|}{$\begin{array}{l}\text { Primary cardiac } \\
\text { malformation }\end{array}$} \\
\hline Tricuspid atresia & $5(22)$ & $5(50)$ & $10(30)$ \\
\hline Double inlet LV & $6(26)$ & $3(30)$ & $9(28)$ \\
\hline Double outlet RV & $4(18)$ & $1(10)$ & $5(15)$ \\
\hline Complete AVSD & $3(13)$ & $0(0)$ & $3(9)$ \\
\hline Pulmonary atresia & $2(9)$ & $1(10)$ & $3(9)$ \\
\hline Mitral valve atresia & $1(4)$ & $0(0)$ & $1(3)$ \\
\hline Ebstein anomaly & $1(4)$ & $0(0)$ & $1(3)$ \\
\hline Criss-cross ventricles & $1(4)$ & $0(0)$ & $1(3)$ \\
\hline \multicolumn{4}{|l|}{ Fontan connection } \\
\hline Atriopulmonary Fontan & $20(88)$ & $8(80)$ & $28(85)$ \\
\hline Lateral tunnel Fontan & $1(4)$ & $1(10)$ & $2(6)$ \\
\hline Right atrium to RVOT & $1(4)$ & $1(10)$ & $2(6)$ \\
\hline Extracardiac conduit & $1(4)$ & $0(0)$ & $1(3)$ \\
\hline
\end{tabular}

sinusoidal fibrosis, the time of death ranged from 34 days to 25.6 years after the Fontan operation. All the patients with grade 4 sinusoidal fibrosis had undergone the Fontan operation at an older age (range, 14-34.5 years) and had left ventricular morphology. Four of these patients had undergone previous staging with a cavopulmonary shunt. In a multivariate model, the interval from the Fontan operation to death was related to the degree of sinusoidal fibrosis $(P=.03)$ but age at the Fontan operation was not $(P=.06)$.

TABLE 2. Operations before Fontan procedure

\begin{tabular}{lccc}
\hline \multicolumn{1}{c}{ Operation } & $\begin{array}{c}\text { Early deaths } \\
(\mathbf{n}=\mathbf{2 3})\end{array}$ & $\begin{array}{c}\text { Late deaths } \\
(\mathbf{n}=\mathbf{1 0})\end{array}$ & $\begin{array}{c}\text { Total deaths } \\
(\mathbf{n}=\mathbf{3 3})\end{array}$ \\
\hline $\begin{array}{l}\text { Pulmonary artery band } \\
\text { Pulmonary artery band }\end{array}$ & $3(13)$ & $1(10)$ & $4(12)$ \\
$\quad 2(9)$ & $0(0)$ & $2(6)$ \\
and atrial septectomy & & & \\
Blalock-Taussig shunt & $9(39)$ & $5(5)$ & $14(42)$ \\
Aortopulmonary shunt & $5(22)$ & $3(30)$ & $8(24)$ \\
Glenn anastomosis & $1(4)$ & $1(10)$ & $2(6)$ \\
Atrial septectomy only & $1(4)$ & $0(0)$ & $1(3)$ \\
TV replacement and & $1(4)$ & $0(0)$ & $1(3)$ \\
$\quad$ Glenn anastomosis & & & \\
None & $1(4)$ & $0(0)$ & $1(3)$ \\
\hline
\end{tabular}

Data presented as n (\%). TV, Tricuspid valve.

\section{Portal Fibrosis}

Portal fibrosis, as determined by the Ishak ${ }^{11}$ and Scheuer ${ }^{12}$ scoring systems was demonstrated in 17 of 33 patients $(52 \%$; Table 3). Fewer patients had portal fibrosis $(52 \%)$ than sinusoidal fibrosis $(76 \%)$. In the total cohort, 6 of 33 patients $(18 \%)$ had an Ishak score of grade 3 or greater and 9 of 33 patients $(27 \%)$ had a modified Scheuer score of grade 2 or greater (Table 3). The degree of portal fibrosis correlated directly with the time from the Fontan operation $(P<.002$; Figure $1, C$ and $D$, and Figure 2, $C)$. Patients who died more than 5.1 years after the Fontan operation had significant portal fibrosis using both grading scores (Figure 2, $C)$. However, 1 patient, who had died unexpectedly of a pulmonary embolism, had minimal portal fibrosis 7.4 years after the Fontan operation.

\section{Additional Variables}

The additional patient variables analyzed included gender, ventricular morphology, history of heterotaxy syndrome, history of PLE, and Fontan connection type. None of these variables were significantly associated with the degree of hepatic congestion, portal fibrosis, or sinusoidal fibrosis. Catheterization data from before the Fontan operation were available for a limited number of patients. The ventricular end-diastolic pressure ranged from 5 to $30 \mathrm{~mm} \mathrm{Hg}$ for 17 of 33 patients (52\%) and had no significant relationship with the degree of hepatic fibrosis.

\section{Early Deaths After Fontan Operation}

Of the total cohort, 23 of 33 patients $(70 \%)$ died within 35 days of the Fontan operation. Sinusoidal fibrosis occurred in 15 of the 23 patients $(65 \%)$ who died early. Of these 15 patients with sinusoidal fibrosis, $10(67 \%)$ had left ventricular morphology and $6(40 \%)$ had heterotaxy syndrome. Sinusoidal fibrosis grade 2 or greater was seen in 7 patients in the 15-patient subset, and 4 of these 7 patients $(57 \%)$ had heterotaxy syndrome.

In these 23 patients, portal fibrosis was observed in only 7 $(30 \%)$ using both the Ishak ${ }^{11}$ and modified Scheuer ${ }^{12}$ grading scores (Table 3). Of these 7 patients with portal fibrosis, $4(57 \%)$ had heterotaxy syndrome and $6(86 \%)$ had single ventricles with right ventricular morphology. Only 2 patients in this subset had grade 2 portal fibrosis, and both of these patients had heterotaxy syndrome.

\section{DISCUSSION}

Hepatic disease has been increasingly recognized in patients after the Fontan operation and contributes to the morbidity and mortality of these patients. The present autopsy series represents the largest histologic analysis of liver specimens in patients after the Fontan operation to date. We have shown that the severity of both sinusoidal fibrosis and portal fibrosis increases with time after the Fontan operation 

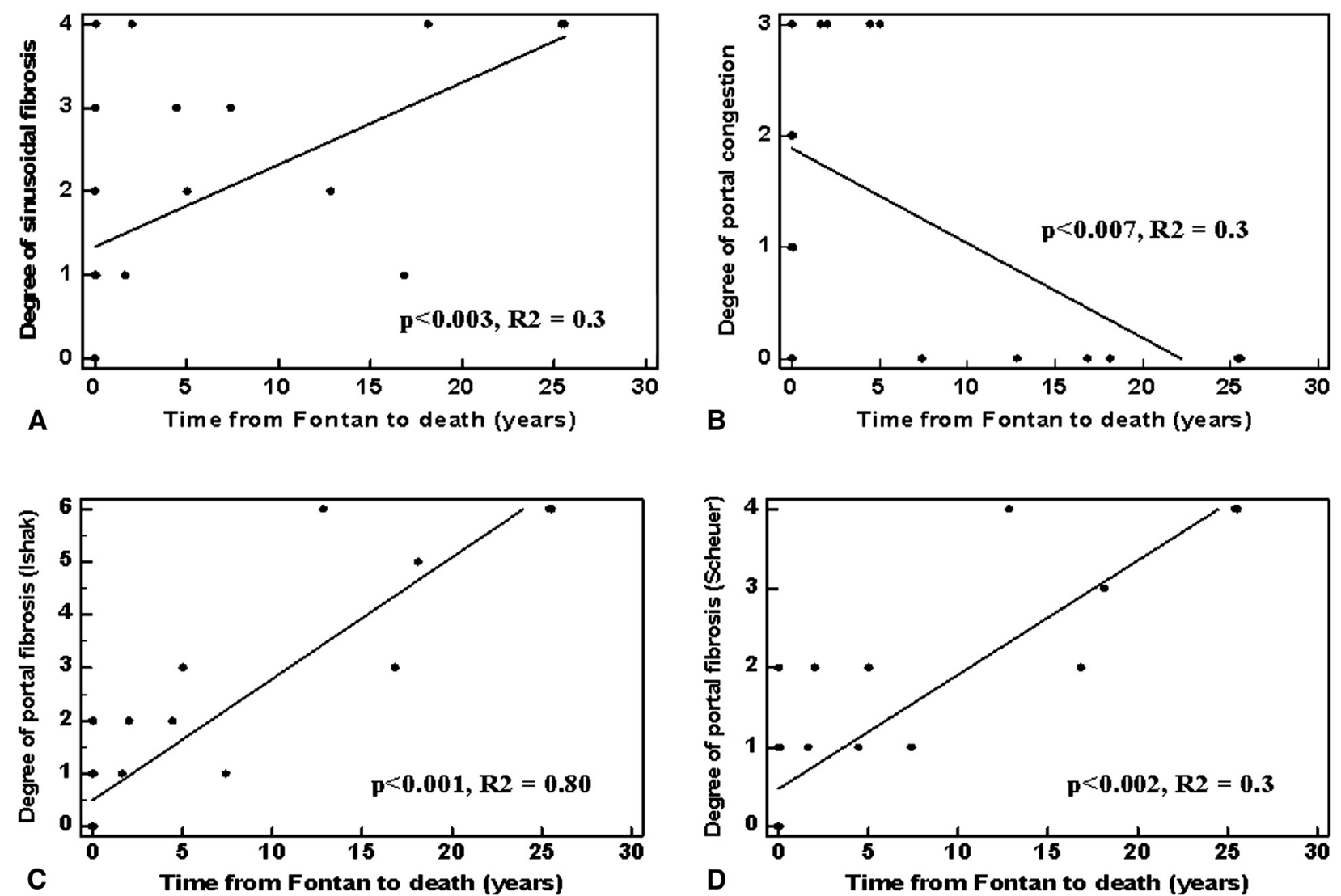

FIGURE 1. Scatter plots of interval from Fontan operation to death showing degree of liver disease correlated with interval from Fontan operation to death. Sinusoidal fibrosis, portal fibrosis, and hepatic congestion graded per standard histologic grading scales. ${ }^{11,12}$ Simple linear regression analysis of (A) sinusoidal fibrosis, (B) portal congestion, (C) portal fibrosis by Ishak grade, and (D) portal fibrosis by Scheuer grade and interval from Fontan operation to death.

(Figure 2, $B$-D). In addition, the severity of sinusoidal fibrosis correlated with older age at the Fontan operation, which has not been previously reported. Significant sinusoidal fibrosis was observed in patients who died early $(<35$ days $)$ after the Fontan operation, with more severe disease occurring in patients with heterotaxy syndrome.

Sinusoidal fibrosis is more common in venous outflow impairment in which elevated central venous pressure results in fibrosis around the central vein extending out to the hepatic sinusoids. Sinusoidal fibrosis in patients after the Fontan operation is, in part, thought to be due to exposure to high pressure within the Fontan pathway and intrahepatic reflux from right atrial contraction in those patients with an atriopulmonary Fontan. ${ }^{5,6}$ Typically, portal fibrosis is associated with inflammatory diseases of the liver; however, portal fibrosis can also occur with longstanding venous outflow impairment. ${ }^{13}$ Venous outflow impairment can mimic biliary disease in biopsy specimens,

TABLE 3. Degree of fibrosis stratified by time of death

\begin{tabular}{|c|c|c|c|c|c|c|c|c|c|}
\hline \multirow[b]{2}{*}{$\begin{array}{c}\text { Fibrosis } \\
\text { degree }\end{array}$} & \multicolumn{3}{|c|}{ Sinusoidal fibrosis } & \multicolumn{3}{|c|}{ Ishak grade of portal fibrosis } & \multicolumn{3}{|c|}{ Scheuer grade of portal fibrosis } \\
\hline & $\begin{array}{c}\text { Early death } \\
(\mathbf{n}=\mathbf{2 3})\end{array}$ & $\begin{array}{c}\text { Late death } \\
(\mathbf{n}=10)\end{array}$ & $\begin{array}{c}\text { Total } \\
(\mathbf{n}=\mathbf{3 3})\end{array}$ & $\begin{array}{l}\text { Early death } \\
(\mathrm{n}=\mathbf{2 3})\end{array}$ & $\begin{array}{c}\text { Late death } \\
(\mathbf{n}=10)\end{array}$ & $\begin{array}{c}\text { Total } \\
(\mathbf{n}=\mathbf{3 3})\end{array}$ & $\begin{array}{l}\text { Early death } \\
(\mathbf{n}=\mathbf{2 3})\end{array}$ & $\begin{array}{c}\text { Late death } \\
(\mathbf{n}=\mathbf{1 0})\end{array}$ & $\begin{array}{c}\text { Total } \\
(\mathbf{n}=\mathbf{3 3})\end{array}$ \\
\hline 0 & 8 & 0 & 8 & 16 & 1 & 17 & 16 & 0 & 16 \\
\hline 1 & 8 & 2 & 10 & 5 & 1 & 6 & 5 & 3 & 8 \\
\hline 2 & 2 & 2 & 4 & 2 & 2 & 4 & 2 & 3 & 5 \\
\hline 3 & 4 & 2 & 6 & 0 & 2 & 2 & 0 & 1 & 1 \\
\hline 4 & 1 & 4 & 5 & 0 & 0 & 0 & 0 & 3 & 3 \\
\hline 5 & & & & 0 & 1 & 1 & & & \\
\hline 6 & & & & 0 & 3 & 3 & & & \\
\hline
\end{tabular}




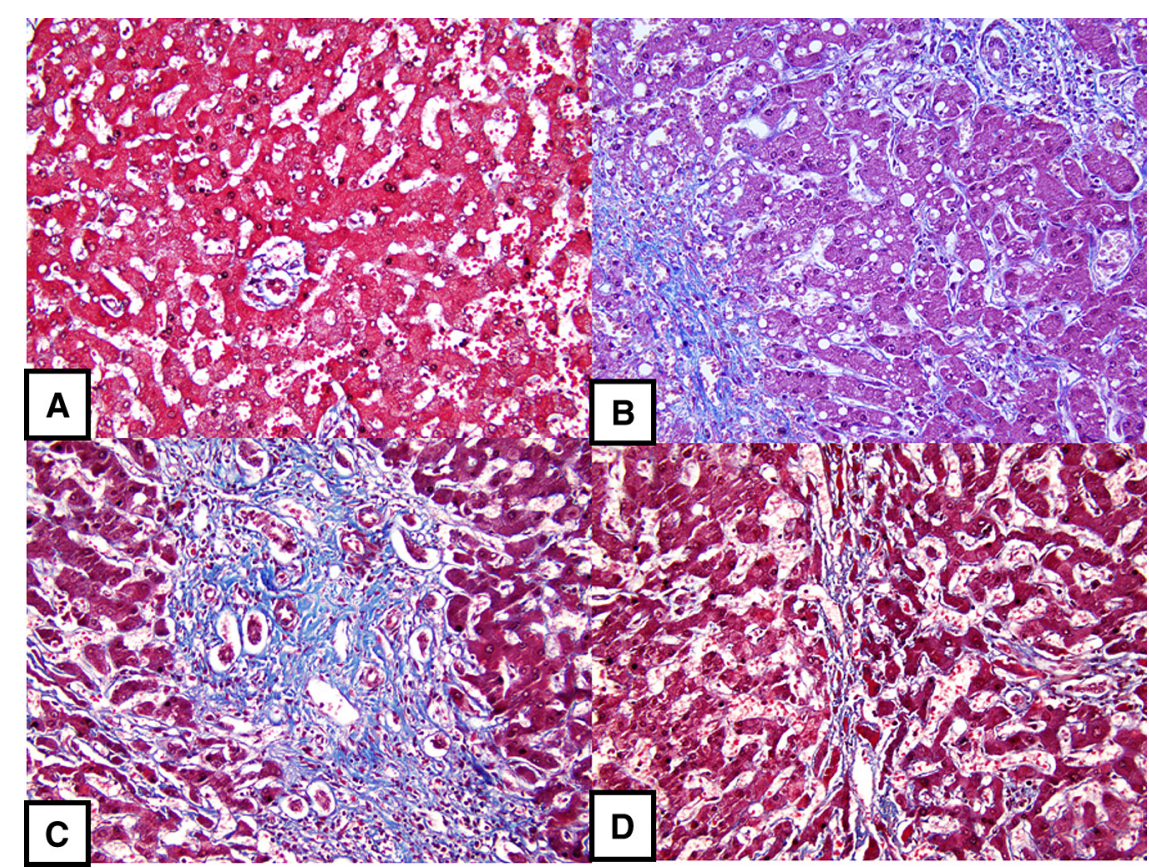

FIGURE 2. A, Medium-magnification photomicrograph showing normal liver histopathologic features with absent portal fibrosis and sinusoidal fibrosis (Masson trichrome stain) in a patient who died 1 day after Fontan procedure. B, Medium-magnification photomicrograph showing severe (grade 4/4) sinusoidal fibrosis (Masson trichrome stain) in a patient who died 34 days after Fontan procedure. High-power views of (C) marked portal fibrosis with bile ductal proliferation and (D) marked grade (4/4) sinusoidal fibrosis in a patient who died 25.6 years after Fontan procedure.

because it can produce, not only portal and periportal fibrosis, but also ductular proliferation.

Cirrhosis due to cardiac disease has been associated with a variety of cardiac conditions, including acute and chronic cardiac failure. ${ }^{14,15}$ Chronic elevation of the right atrial pressure is thought to result in sinusoidal congestion and fibrosis. ${ }^{15}$ After the Fontan operation, elevated systemic venous pressure, decreased cardiac output, decreased heart rate, and longer follow-up after the Fontan operation have been associated with the development of hepatic disease. ${ }^{7,16}$ Both sinusoidal fibrosis and portal fibrosis have been reported in this patient population, with a recent report citing liver disease occurring before the Fontan operation as a result of pre-Fontan morbidity. ${ }^{5-8}$

Similar to previous studies, we found that a longer interval from the Fontan operation to death correlated with increased severity of both portal and sinusoidal fibrosis. In our cohort, sinusoidal fibrosis occurred more frequently than portal fibrosis $(76 \%$ vs $51 \%)$. An interesting, previously unreported, finding was that older age at the initial Fontan operation correlated with a greater grade of sinusoidal fibrosis. In general, the patients in our study were an older at the Fontan operation (median age, 6.2 years; range, 1.9-35.9 years) compared with the current standard operation timing. In the patients with the greatest grade of sinusoidal fibrosis, a common denominator was older age at the initial Fontan operation (range, 14-34.5 years).
Clinically, 4 patients who died late after the Fontan operation had symptoms of ascites; all had died of multiorgan system failure occurring either postoperatively or secondary to sepsis. However, 3 of these patients also had PLE. The remaining 6 patients did not show overt clinical signs of liver dysfunction, and the serum liver function test results were normal. This supports our previously published data from patients with PLE after the Fontan operation. Often, the serum laboratory assessment results of the liver are normal for these patients; however, the patients can demonstrate signs of hepatic dysfunction during signs of stress such as surgery or infection. ${ }^{17}$ In addition, medications that are normally cleared through the liver can have delayed clearance and increased side effects.

Sinusoidal fibrosis was also seen in 15 of the 23 patients $(65 \%)$ who died early after the Fontan operation, implying that these changes had occurred before the Fontan operation. Seven of these patients had significant sinusoidal fibrosis (grade 2 or greater). These findings likely resulted from an elevation in right atrial pressure. Delaying the Fontan operation might not necessarily prevent hepatic fibrosis, because greater grades of both sinusoidal congestion and sinusoidal fibrosis were seen in the patients who had undergone the Fontan operation at an older age. Because our sample size was limited, additional data are needed to substantiate this finding.

Recent reports have shown that portal fibrosis can occur before the Fontan operation as a result of pre-Fontan 
morbidity. Schwartz and colleagues ${ }^{8}$ reported that $86 \%$ of patients (most with hypoplastic left heart syndrome) had portal fibrosis before the Fontan operation. In contrast to that study, we did not find severe portal fibrosis in patients who died early after the Fontan operation, implying portal fibrosis was not present before the initial Fontan operation in our cohort (Figure 1,A). Only 7 of the patients who died early in our cohort had any portal fibrosis, and only 2 of these patients had grade 2 fibrosis on the Scheuer ${ }^{12}$ grading scale. Given the era of surgery, certainly, inflammatory hepatopathies could also have contributed to these findings. Only $15 \%$ of patients had documented negative hepatitis C test results; however, no evidence of inflammation was seen on the histologic assessment that would have been indicative of inflammatory hepatopathy. The mild fibrosis seen in our patient population could have resulted from longstanding, high, right atrial pressures preoperatively, because most patients underwent their Fontan operations at an older age.

Heterotaxy syndrome did not correlate with the presence of hepatic disease in our cohort. However, patients with heterotaxy syndrome had a greater severity of hepatic disease than those who died early after the Fontan operation. Only 2 patients had grade 2 portal fibrosis in this subset; both of these patients had heterotaxy syndrome (asplenia). Of the 7 patients with grade 2 sinusoidal fibrosis or greater, 4 $(57 \%)$ had heterotaxy syndrome, 3 of whom had asplenia. Patients with heterotaxy syndrome will have comorbidity such as biliary atresia. Increased hepatic fibrosis has not been previously described in this patient population. The subtype of heterotaxy might contribute to the disease severity; however, because the numbers were quite small, this requires additional study. We also studied 6 patients with heterotaxy syndrome who died before their Fontan operation. Of these patients, only 1 had grade 1 portal fibrosis, and none had sinusoidal fibrosis. Thus, it is unclear whether significant sinusoidal or portal fibrosis occurs in patients with heterotaxy syndrome and a functional single ventricle before the Fontan operation.

\section{Study Limitations}

The present study was a small, retrospective study. Many patients had undergone primary palliative operations at other institutions, and the pre-Fontan operative history and cardiac catheterization data were not always available. The patients in our cohort tended to have undergone the Fontan operations at an older age with predominantly atriopulmonary Fontan connections.

\section{CONCLUSIONS}

The incidence of hepatic fibrosis increased proportionally with patient age at the Fontan operation, placing these patients at serious risk of liver disease. The interval from the Fontan operation to death was associated with increased sinusoidal fibrosis and portal fibrosis. However, the adverse liver changes appeared to occur even before the Fontan operation and correlated with older age at the Fontan operation. Heterotaxy syndrome, although not statistically significant, was a feature seen in many patients with more severe portal and sinusoidal fibrosis before the Fontan operation.

\section{References}

1. Fontan F, Baudet E. Surgical repair of tricuspid atresia. Thorax. 1971;26:240-8

2. Cohen AJ, Cleveland DC, Dyck J, Poppe D, Smallhorn J, Freedom RM, et al. Results of the Fontan procedure for patients with univentricular heart. Ann Thorac Surg. 1991;52:1266-71.

3. Lemmer JH, Coran AG, Behrendt DM, Heidelberger KP, Stern AM. Liver fibrosis (cardiac cirrhosis) 5 years after modified Fontan operation for tricuspid atresia. J Thorac Cardiovasc Surg. 1983;86:757-60.

4. Khairy P, Poirier N, Mercier LA. Univentricular heart. Circulation. 2007;115 800-12.

5. Ghaferi AA, Hutchins GM. Progression of liver pathology in patients undergoing the Fontan procedure: chronic passive congestion, cardiac cirrhosis, hepatic adenoma, and hepatocellular carcinoma. J Thorac Cardiovasc Surg. 2005;129: 1348-52.

6. Kendall TJ, Stedman B, Hacking N, Haw M, Vettukattill JJ, Salmon AP, et al Hepatic fibrosis and cirrhosis in the Fontan circulation: a detailed morphological study. J Clin Pathol. 2008;61:504-8.

7. Kiesewetter CH, Sheron N, Vettukattill JJ, Hacking N, Stedman B, MillwardSadler H, et al. Hepatic changes in the failing Fontan circulation. Heart. 2007; 93:579-84.

8. Schwartz MC, Sullivan L, Cohen MS, Russo P, John AS, Guo R, et al. Hepatic pathology may develop before the Fontan operation in children with functional single ventricle: an autopsy study. J Thorac Cardiovasc Surg. 2012;143:904-9.

9. Stanton RE, Lurie PR, Lindesmith GG, Meyer BW. The Fontan procedure for tricuspid atresia. Circulation. 1981;64(2 Pt 2):II140-6.

10. Saliba T, Dorkhom S, O'Reilly EM, Ludwig E, Gansukh B, Abou-Alfa GK. Hepatocellular carcinoma in two patients with cardiac cirrhosis. Eur J Gastroenterol Hepatol. 2010;22:889-91.

11. Ishak K, Baptista A, Bianchi L, Callea F, De Groote J, Gudat F, et al. Histological grading and staging of chronic hepatitis. J Hepatol. 1995;22:696-9.

12. Scheuer PJ. Classification of chronic viral hepatitis: a need for reassessment. J Hepatol. 1991;13:372-4.

13. Kakar S, Batts KP, Poterucha JJ, Burgart LJ. Histologic changes mimicking biliary disease in liver biopsies with venous outflow impairment. Mod Pathol. 2004; 17:874-8.

14. Lefkowitch JH, Mendez L. Morphologic features of hepatic injury in cardiac disease and shock. J Hepatol. 1986;2:313-27.

15. Myers RP, Cerini R, Sayegh R, Moreau R, Degott C, Lebrec D, et al. Cardiac hepatopathy: clinical, hemodynamic, and histologic characteristics and correlations. Hepatology. 2003;37:393-400.

16. Camposilvan S, Milanesi O, Stellin G, Pettenazzo A, Zancan L, D'Antiga L. Liver and cardiac function in the long term after Fontan operation. Ann Thorac Surg. 2008;86:177-82.

17. John AS, Driscoll DJ, Warnes CA, Phillips SD, Cetta F. The use of oral budesonide in adolescents and adults with protein-losing enteropathy after the Fontan operation. Ann Thorac Surg. 2011;92:1451-6. 